\title{
The International Journal of Orthodontia
}

Editor: Martin Dewey, D.D.S., M.D.

VOL. III

St. Louis, August, 1917

No. 8

\section{ORIGINAL ARTICLES}

\section{THE RELATION BETWEEN MALOCGLUSION AND NASAL DEFORMITIES}

By Martin Dewey, D.D.S., M.D., Chicago, Ill.

$\mathrm{T}^{\mathrm{T}}$ has long been recognized that there is a relation existing between maloc1 clusion of the teeth and deformities of the nasal cavity, but there has been some dispute in regard to what relation one has to the other and which is the causative factor and which is the effect. In considering the etiology of malocclusion, we have learned that a certain number of malocclusions are caused by mouth breathing which is produced by abnormal forces of occlusion resulting from a disturbed function of the muscle and abnormal atmospheric pressure. It has also been observed that certain types of nasal deformities are almost invariably associated with certain types of malocclusion, and it has long been recognized that many of these types of nasal deformities can not be improved to any great extent unless the treatment is associated with the correction of the malocclusion.

There are a number of individuals who are suffering from mouth breathing in whom the mouth breathing has undoubtedly been caused by hypertrophy of the lymphoid tissue in the nasopharynx and upon removal of this lymphoid tissue the mouth breathing still persists. This is because the abnormal action of the muscles and abnormal atmospheric pressure during the time the individual has been a mouth breather has produced a malocclusion and along with the malocclusion has been produced a deformity or abnormal development of the nasal cavity. We are then naturally confronted by the question as to what relation exists between malocclusion of the teeth and deformed nasal cavities, which is the causative factor, and which is the effect, and what benefit can be expected to be derived from the correction of malocclusion so far as the deformed nasal cavity is concerned. Considering the relation between malocclusion and deformed nasal cavities, it is well to remember the anatomy of the 
parts concerned. It must be remembered that the nasal and oral cavity as they are found in the adult have at one time been a common cavity which may be described from an embryological standpoint as consisting of a single opening at the anterior end of the alimentary canal which is known as a stomodæum. It must be remembered in the early life of the individual that the nasal and oral cavities are one cavity without any separating structure, or, in other words, the

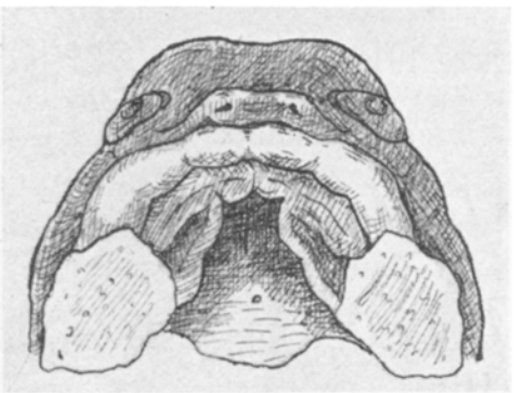

Fig. 1.-Drawing showing embryonic development of roof of mouth.

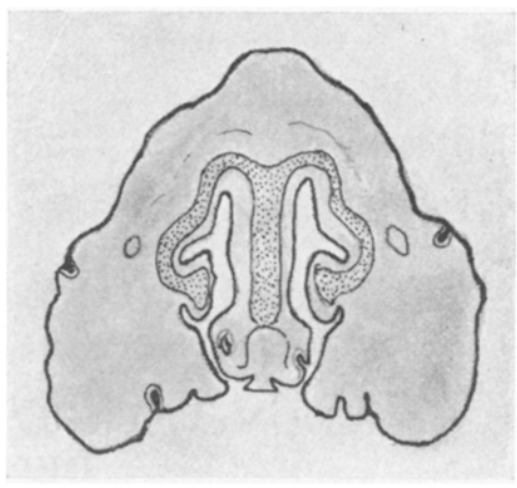

Fig. 2.-Cross section of developing nasal and oral cavity of embryo pig. (After His.)

hard and soft palates have not yet developed to separate the nasal from the oral cavity.

In the development of the nasal and oral cavity we find the first branchial arch and the fronto-nasal process play a very important part. The first branchial arch divides into two parts, one of which is known as the mandibular portion, and is the lower part; and the upper portion known as the maxillary portion

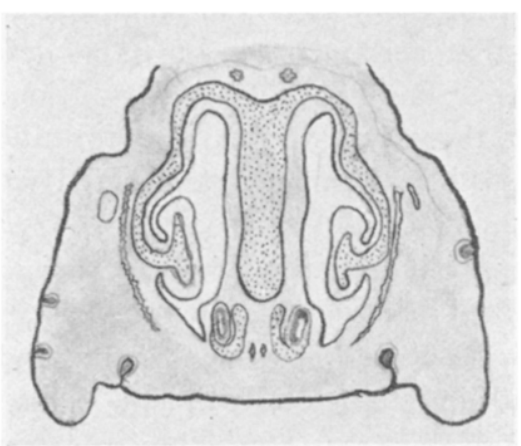

Fig. 3.-Cross section of developing nasal cavity after the union of the palatal processes of the superior
maxilla. (After His.)

which grows from the superior right and left side of the mandibular arch and finally unites with the frontal-nasal bud in that region which may be compared to the lip or alveolar border. From the inner posterior portion of the first branchial arch there grows a shelf or offshoot which finally unites in the median line which forms the hard and soft palate. It is a formation of the hard and soft palate which separates the nasal from the oral cavity. 
The developments of these parts can be observed in Fig. 1, which is copied after His. Another structure or development which must be considered in relation to malocclusion and deformity of the nasal cavity is the development of the nasal septum, the turbinated body shown in Fig. 2. Growing downward from the base of the cranium, or from the chondro-cranium, is a cartilaginous structure or rather structures which make up or contribute to what is known in later life as the nasal septum. Growing down from the horizontal plate of the ethmoid is the perpendicular plate of the ethmoid, which grows downward into the nasal cavity towards the median line or the junction of the hard palate. From the base of the sphenoid we find another cartilaginous structure which grows forward and which is known as the cartilaginous vomer. It is later replaced by intramembranous bones developing on the right and left sides of the cartilage. Associated with the perpendicular plate of the ethmoid and the vomer we have

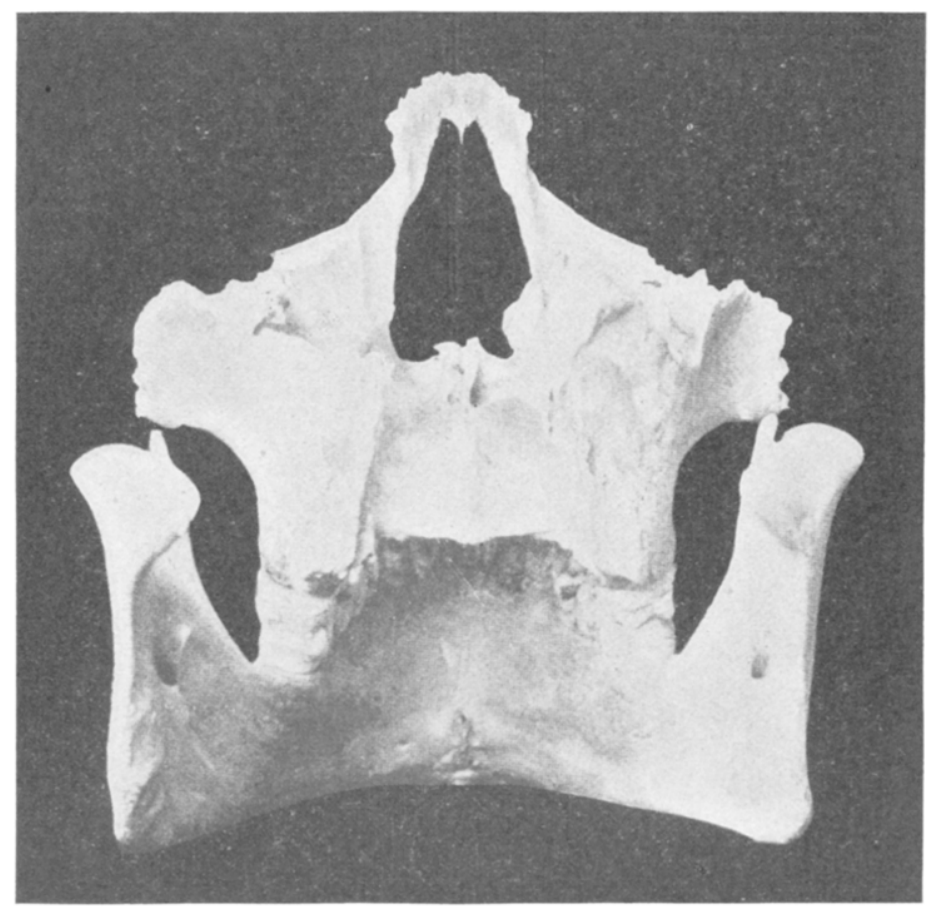

Fig. 4.--Posterior view of nasal cavity showing bony cavity which contains the nasal septum.

a cartilage known as the triangular cartilage which completes what is later known in life as the nasal septum. The downward growth of the perpendicular plate of the ethmoid and the vomer occurs regardless of the development of the lateral halves of the nasal cavity. They occur regardless of the development of. the floor of the nose and it is this factor which must be kept in mind in considering the relation of the deformity of the nasal cavity in relation to malocclusion. As the nasal septum grows down from the chondro-cranium the horizontal plate of the superior maxillary bone, which has been developed in the maxillary bud of the first branchial arch grows towards the median line and the structures unite at the median line forming the floor of the nose, the nose being 
divided by the nasal septum into the right and left nares. The embryonic development of the nasal septum and the floor of the nose is shown in Fig. 3.

Fig. 4 is a posterior view of the maxillary bone and the mandible showing the bony wall of the oral cavity and the nasal cavity as viewed from the posterior border. It will be seen that the hard palate or the roof of the mouth also forms the floor of the nose. The right and left halves of the nares are made up by the perpendicular plate of the superior maxillary bone or the nasal process of the superior maxillary, while the superior lateral walls of the nose are made up of the lateral masses of the ethmoid. The horizontal plate of the ethmoid assisted by a small portion of the frontal and body of the sphenoid bone forms the roof of the nose. Thus the nasal cavity is formed, completely surrounded by a bony structure and divided by the nasal septum. The nasal cavity is separated into the right and left nares by the nasal septum which has grown down from the perpendicular plate of the ethmoid, the body of the sphenoid, and which grows down toward the roof of the mouth in the median line. The nasal

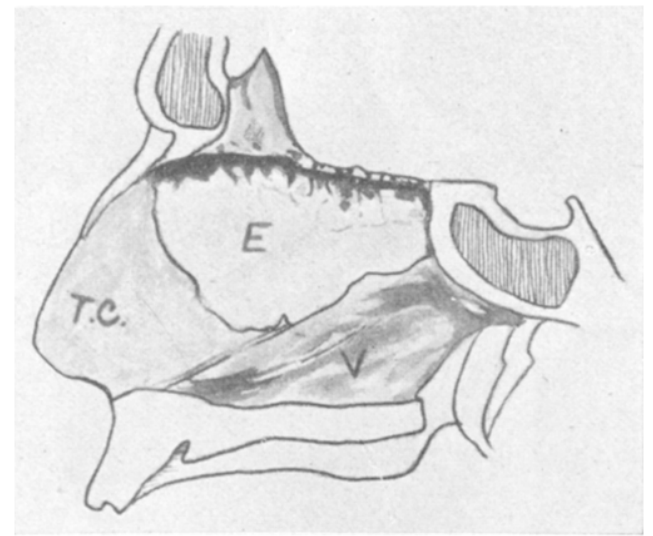

Fig. 5.--Diagram of nasal septum. T.C., Triangt1lar cartilage; E., Perpendicular plate of ethmoid; V., Vomer.

septum is primarily of cartilaginous origin and is replaced by an osseous structure. Physiologically the nasal septum has nothing to do with respiration. From an evolutionary standpoint the vomer was originally al tooth-bearing bone and has been walled off in the nasal cavity during the process of evolution.

As we study this question further we find that the factors which affect the development of the lateral walls of the nasal cavity and the floor of the nasal cavity do not necessarily affect the septum. As a result of this, there are certain types of malocclusion in which the nasal cavity does not develop as rapidly as it should. The nasal septum will continue to grow downward and meeting with resistance from the floor of the nose, will become deflected. Fig. 5 is a diagram showing the various parts of the nasal septum, of which T.C is the triangular cartilage, $E$ is the perpendicular part of the ethmoid, and $V$ is the vomer. It will be observed that the vomer grows downward and forward originally in cartilage and is later replaced by intermembranous bony development on both sides of the cartilage and the cartilage disappears. As a result of this, the vomer may be considered a double structure composed of a right and a left 
side. The perpendicular plate of the ethmoid grows downward from the horizontal plate of the ethmoid and joins the vomer and the triangular cartilage. The anterior portion of the nasal septum is completed by the triangular cartilage which is also subject to a great many deformations which may be the result of abnormal development of the nasal cavity as the result of injury. Carefully. studying Fig. 5 you will see the nasal septum is enclosed between two bony walls or surfaces which are the roof of the nose and the floor of the nose. The distance between the floor of the nose and the roof of the nose is dependent upon the development of the lateral walls of the nose, the principal portion of which is the superior maxillary bone. Therefore, if any condition arises that inter-

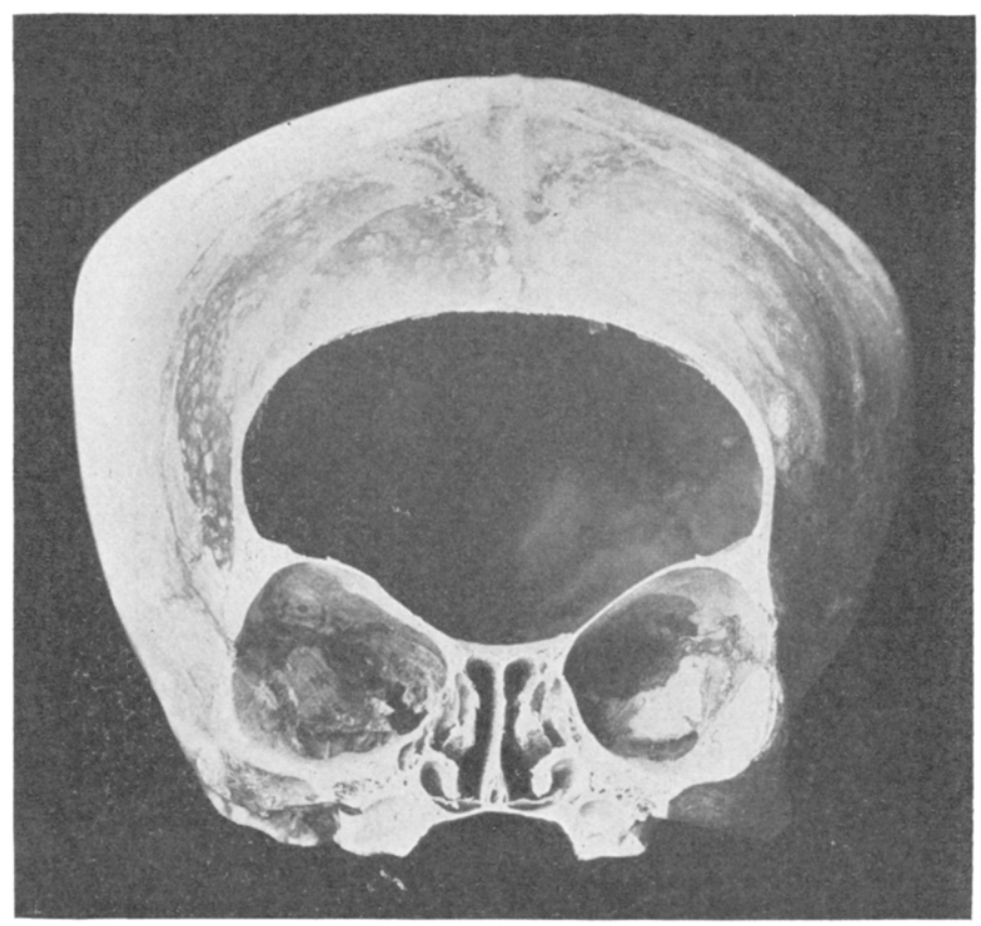

Fig. 6.-Nasal septum enclosed in nasal cavity.

feres with the growth of the superior maxillary bone it necessarily will cause a shortness between the floor of the nose and the roof of the nose. The nasal septum will be compelled to occupy a smaller space than it was originally intended to occupy.

It is also a fact that conditions which affect the development of the lateral wall of the nose do not affect the nasal septum. Therefore, in a great many types of malocclusion we find that deflected nasal septi are the result of the lack of development of the lateral walls of the nasal cavity and the superior maxillary bone which have resulted in a lack of distance between the roof and the floor of the nose. Fig. 6 shows a condition of a fetus skull at birth and it will be observed at this time that the bony septum or nasal septum is enclosed in the bony walls of the nasal cavity. It will also be observed that the roof of the mouth is comparatively flat, that the alveolar process is close to the 
orbital cavity, and that there must be great increase in distance between the floor of the orbital cavity and the alveolar process as the individual develops. The inferior turbinated bone is very close to the roof of the mouth or floor of the nose with the result that as the individual grows the nasal cavity increases in length especially between the inferior turbinated bone and the floor of the nose.

As this floor of the nose is carried down by the development of the lateral walls, space is made for the nasal septum and if development goes on harmon-

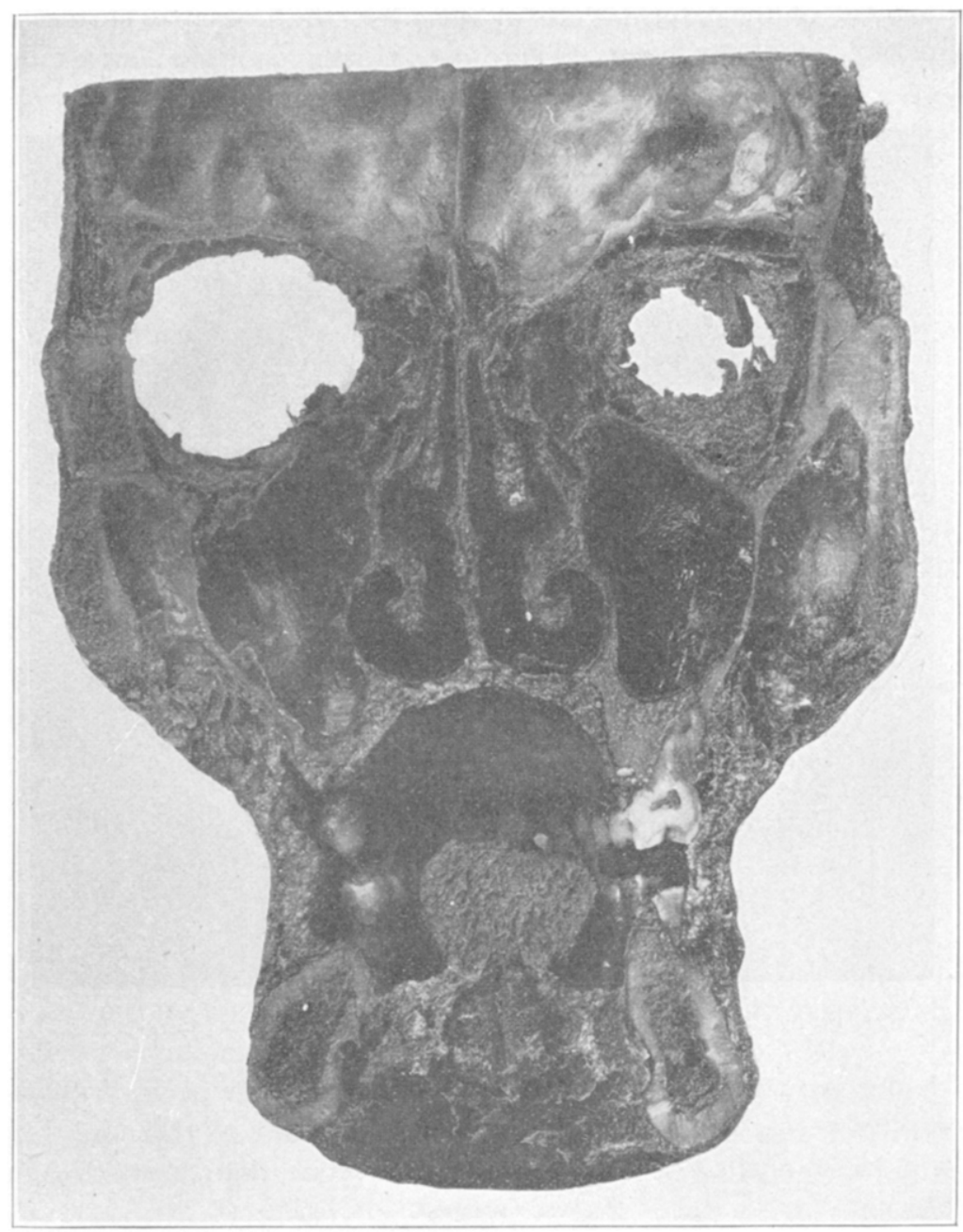

1iig. 7.- Nasal cavity of an adult.

iously, we find a development occurring which in the adult is the same as we find in Fig. 7. It will be observed that there is a tendency for the nasal septum to separate into two parts showing the tendency for the organ to be developed into a right and a left portion.

Fig. 8 is the posterior part of the skull shown in Fig. 7 and it shows the shape of the roof of the mouth, which is a beautiful arch, the development of the maxillary sinuses, and the increased distance between the inferior turbinated 
bones and the floor of the nose. This skull may be considered showing a normal development and one in which there is a normal nasal cavity associated with what was a normal occlusion. In certain types of malocclusion which may be neutroclusion or Class I complicated by lack of development of the dental arches, or which may be distal occlusion complicated by labioversion of the upper incisors (Class II, Division 1), or in fact in any individual who is a mouth breather, one is very liable to have an abnormal development of the nasal cavity because of the abnormal development of the superior maxillary bone.

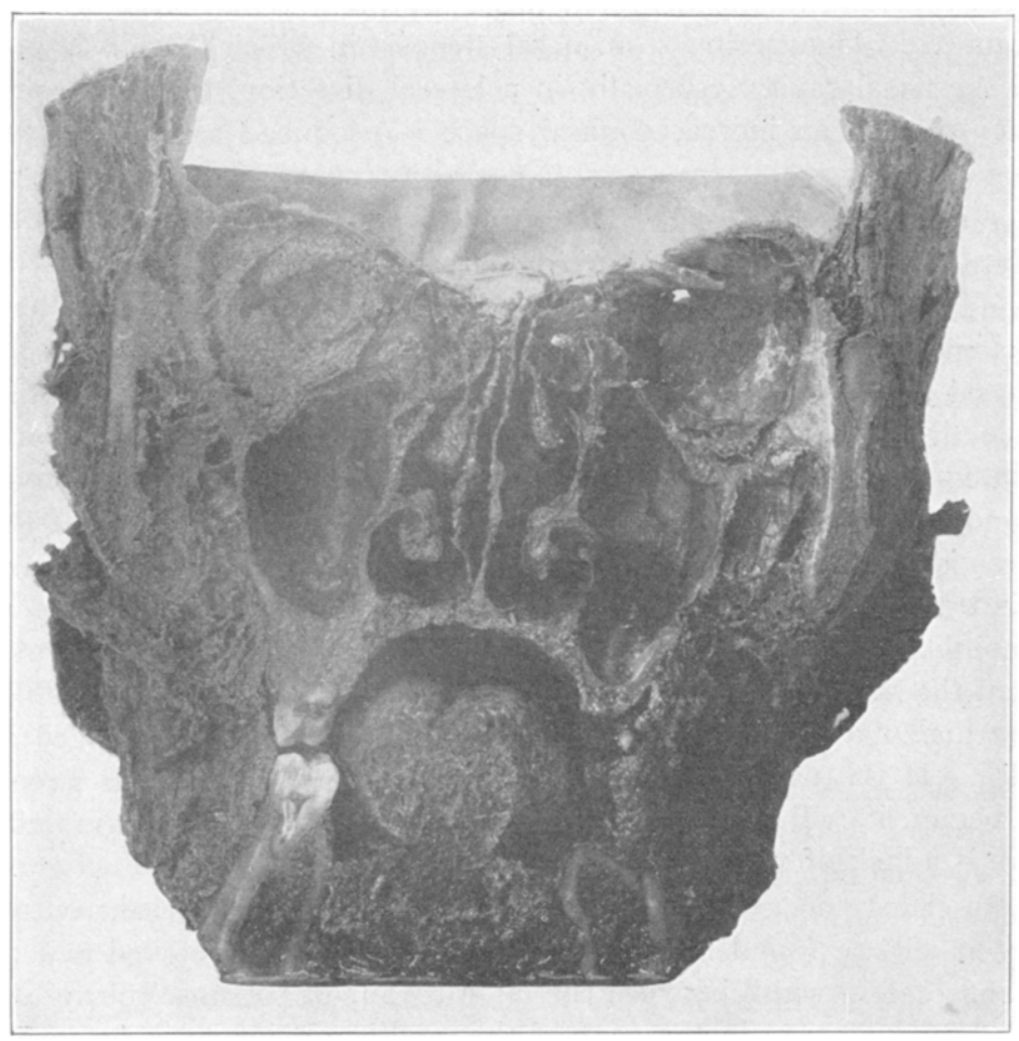

Fig. 8.-Showing double plates of nasal septum.

If the lateral walls of the nasal cavity do not grow down sufficiently rapidly there will be a decrease in distance between the floor of the nose and the roof of the mouth which will result in a deflected septum. The removal of adenoids does not benefit this condition because the only thing that will restore the development of the nasal cavity to the proper size is the treatment of the malocclusion, which will produce the proper growth of the superior maxillary bone and result in changes in the entire nasal cavity. It has long been recognized by a large number of men that the correction of the malocculsion has produced a beneficial result in the deformity or lack of development of the nasal cavity. This observation has led to several plans of treatment, some of which are based 
only on the correction of malocclusion with the resulting change in the nasal cavity while other plans of treatment are based on the correction of the malocclusion with the idea of improving the nasal cavity by changing the shape by mechanical interference.

A number of cases reported several years ago by Ketcham showed that in the treatment of malocclusion in patients who were mouth breathers the nasal cavity increased very materially in size and deflected septi disappeared or improved during the process of treatment. Observations made in my practice have substantiated this fact, so I can state that the correction of the malocclusion in the young individual when the superior dental arch is not properly developed will practically remove the deflected nasal septum. It has also been observed in older individuals suffering from nasal stenosis in which there was insufficient space in the nasal cavity, especially in a lateral direction, that by an expansion of the dental arch an increased nasal space is produced and normal breathing becomes possible.

By study of Figs. 4, 7, or 8 , from a purely mechanical standpoint, it will be seen that any device or kind of treatment which will expand the dental arch and expand the alveolar process of the superior maxillary bone will necessarily produce some change in the width of the nasal cavity. The change in the width of the nasal cavity can be produced in several ways. It must be remembered that the teeth are embedded in the peridental membrane which is a continuation of the muco-periosteum which covers the floor of the nose and the roof of the mouth, and is made up of white inelastic fibers. By an expansion of the clental arch stress is brought to bear upon the fibers of the peridental membrane which in turn is transmitted to the periosteum covering the floor of the nose and roof of the mouth and this exerts an influence upon the floor of the nose to such an extent that the shape of the floor of the nose will be changed and with individuals who have high narrow arches, the roof of the mouth will be changed in height and width. If the nasal cavity is examined in these individuals before treatment is begun, it will be observed that either the nasal septum is deflected or that there is a lack of width between the lateral walls of the nose.

As the change occurs in the roof of the mouth and the nasal cavity is again examined it will be found that the nasal septum has straightened and there has been an increase in width between the lateral walls of the nose. In other words. there has been a change in the roof of the mouth. It has been made wider and the roof of the mouth is lower from the base of the cranium. Some of this change in the roof of the mouth is the direct result of mechanical pressure while more of it is the result of a growth produced by mechanical stimulation and the result of cell metabolism and changes in the structure as a result of natural growth. It must be remembered that the superior maxillary bone is a bone of environment and responds to mechanical influence and stress; and by changing the position of the teeth so as to change the stress upon the bone, the entire superior maxillary bone will be changed in the growing individual. In fact, a great change will also occur in older individuals which has been observed in my practice. The cases which Ketcham reported and the cases observed in my practice were changes which were produced by ordinary orthodontic treatment, which were the result of slow and gradual pressure upon the teeth without any 
direct effort being made to change the shape of the maxillary bone as the result of direct mechanical pressure.

A number of other men have reported cases in which the superior maxillary bone has been changed in shape and in which the nasal cavity has been changed by exerting pressure upon the teeth and the superior maxillary bone with the idea of changing the shape of the roof of the mouth as a result of direct mechanical pressure. This treatment has been based upon the idea that by pressure the median suture between the right and left superior maxillary bone could be opened and as the result of opening this suture a lateral expansion of the nasal cavity would occur.

While I have never followed this plan of treatment in active practice, nevertheless I am convinced that it. is possible to open the intermaxillary suture by the construction of special appliances, and, in fact, it may be done in some cases with any appliance. I have done this experimentally upon dogs at different times without any seemingly ill effect upon the dogs and with the result that the bone has filled in the open suture and the nasal cavity has been increased in width. There is no question but that there are extreme cases of nasal stenosis, narrow dental arches with high palate, in which it is desirable to construct some style of appliance especially suited to widen the lateral halves of the superior maxillary bone, not so much with the idea of opening the median suture as with the idea of producing a bodily movement of the teeth and carrying out the lateral halves of the superior maxillary bone as much as possible. This can be produced by constructing appliances which consist of bands placed upon the molars and the canine teeth, or upon the teeth of the two lateral halves of the arches; and then by the use of alignment wires in such a manner that a direct buccal pressure is exerted upon the teeth, they are forced to move bodily thereby carrying the superior maxillary bone or alveolar process with them in a bodily direction.

In young individuals who have only the deciduous teeth or the deciduous molars and permanent molars, the construction of an appliance for the purpose of producing bodily movement is very unsatisfactory if the appliance is attached directly to the teeth, because it will loosen the deciduous teeth. However, we find that cases at that age are the most desirable for treatment and to overcome the difficulty encountered with loose deciduous teeth the use of the plate has been employed to exert pressure upon the alveolar structures through the soft tissue.

The coffin split plate presented some advantages in the treatment of these cases. The idea has later been modified by other individuals including a device which was published several years ago by Ottolengui, and a modification of the split plate which is used at the present time by Richardson. I have seen a number of cases in Richardson's practice in which this appliance has been used and the results have been very pleasing and satisfactory. All the patients report a great improvement in nasal breathing, improvement in speech, and individuals who before this treatment were only able to breathe through the mouth now breathe through the nose entirely during the day and night.

The appliance, by exerting a gentle pressure on the soft tissues, carries the lateral halves of the superior maxillary bone laterally either by direct mechanical 
influence or by development. The roof of the mouth is changed, the nasal cavity increased in width and deflected nasal septi are straightened because of the increased room that is made for them. I do not believe that the changes which occur in all of these cases are the direct result of mechanical pressure changing the shape of the parts, but are the result of mechanical stimulation of the cells which produces a development which has been retarded and consequently the parts assume a normal proportion and shape resulting in a normal development of the nasal cavity following the correction or treatment of the malocclusion.

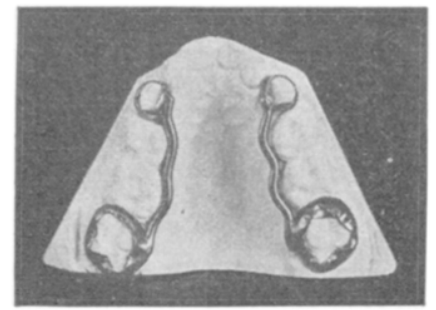

Fig. 9.

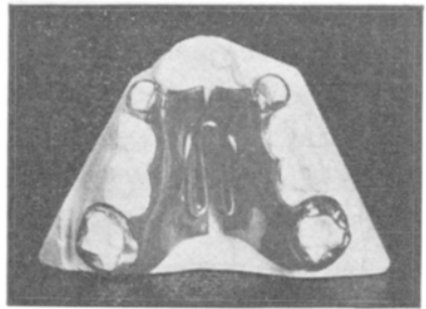

Fig. 10 .

Fig. 9 shows a model of a young patient in whom the only permanent teeth present are the first permanent molars with a high narrow arch. These patients suffer from mouth breathing and invariably show deflected nasal septi and nasal cavities which are below the normal width. The removal of adenoids or tonsils which are usually present does not improve the nasal breathing because of the deformed nasal cavity. The appliance is constructed by making bands upon the permanent molars and the deciduous teeth and adjusting a lingual wire which extends from the canine to the molar bands. This wire should be at least a 16

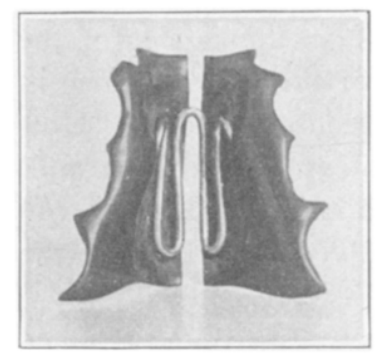

Fig. 11.

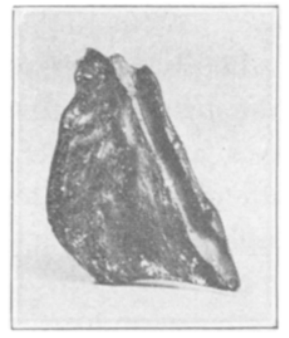

Fig. 12.

gauge wire so as to be of sufficient size to enable the roof plate to grasp the wire securely. After the appliance has been constructed, or rather after the bands have been made for the molars and canines, and the lingual wire is put in position as shown in Fig. 9, the roof plate is made in the ordinary manner of waxing up with base plate wax until it is of the desired thickness. A spring of some sort is embedded in the wax as is shown in Fig. 10 and then the plate is vulcanized in the ordinary manner.

This spring, which is embedded in the roof plate, must be made in the form 
of a W. as shown in Fig. 10 in order to give it a greater range of elasticity and enable it to be so adjusted as to exert a greater expansion either on the canines or molars. If the spring is made in the form of a $W$, it can be opened and greater pressure exerted on the canines than on the molars or can be opened in the posterior border in such a manner as to exert a greater pressure on the molars than on the canines. After the plate is vulcanized it is separated in the middle as shown in Fig. 11 and then by opening the spring the desired amount of pressure can be produced upon the canines or molars.

Fig. 12 shows the palatal view of the plate which is shaped to fit the soft tissue accurately, and pressure will be brought to bear upon the soft tissue and be evenly distributed and produce no soreness. A groove in the gingival border of the plate catches under the lingual wire extending from the canine to the molar and thereby holds it in position. The result produced by this plate is very gratifying because the loose deciduous molars are moved bodily, the roof of the mouth changes shape, the nasal cavity increases in size and the results from the use of this plate could not be surpassed by any other form of appliance. The treatment produces a change in the shape of the roof of the mouth and the shape of the superior maxillary bone and also produces a change in the nasal cavity. With the use of this plate no provision is made to exert pressure upon the anterior teeth if they are protruding and therefore the alignment wire

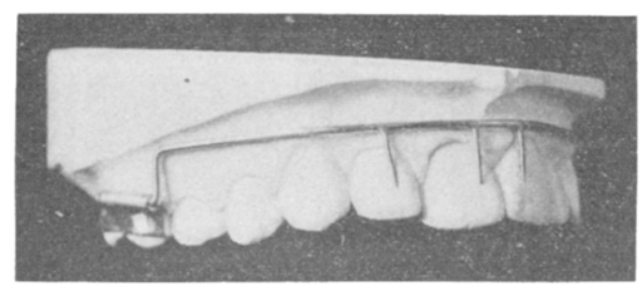

Fig. 13.

must be used to exert pressure on the upper incisors in cases where the upper incisors have erupted.

In young patients in whom the permanent incisors have not erupted, the only kind of treatment necessary at this time is the widening of the superior dental arch so far as the upper teeth are concerned, allowing the deciduous teeth to assume such positions as they will assume as the result of change in the atmospheric and muscular pressure, and the probabilities are, if sufficient space is made for the upper central incisors and the individual is a normal breather at the time they erupt, they will take their normal position. If it is necessary to use an intermaxillary anchorage to correct the mesio-distal length of the arches with the use of this roof plate intermaxillary hooks can be placed on the canine bands to be attached to the lower appliance in the usual manner.

If the patient is more advanced in age and it is desired to bring pressure upon the upper central incisors to correct a protrusion, this can be accomplished by soldering tubes upon the buccal surface of the molar bands which will receive a labial arch as illustrated in Fig. 13. By bending the labial arch gingivally as it leaves the buccal tube it is carried far upon the gingiva and then by using finger spurs pressed against the protruding incisors the appliance is made very 
inconspicuous. The use of the labial arch in no way interferes with the action of the roof plate. The appliance is as inconspicuous as any appliance that can be used. Intermaxillary anchorage can also be employed by having the spurs soldered on the canine bands or by having an intermaxillary hook attached on the labial arch. It would be preferable, in using intermaxillary anchorage, to have the spurs attached to the canine band because that would give a greater

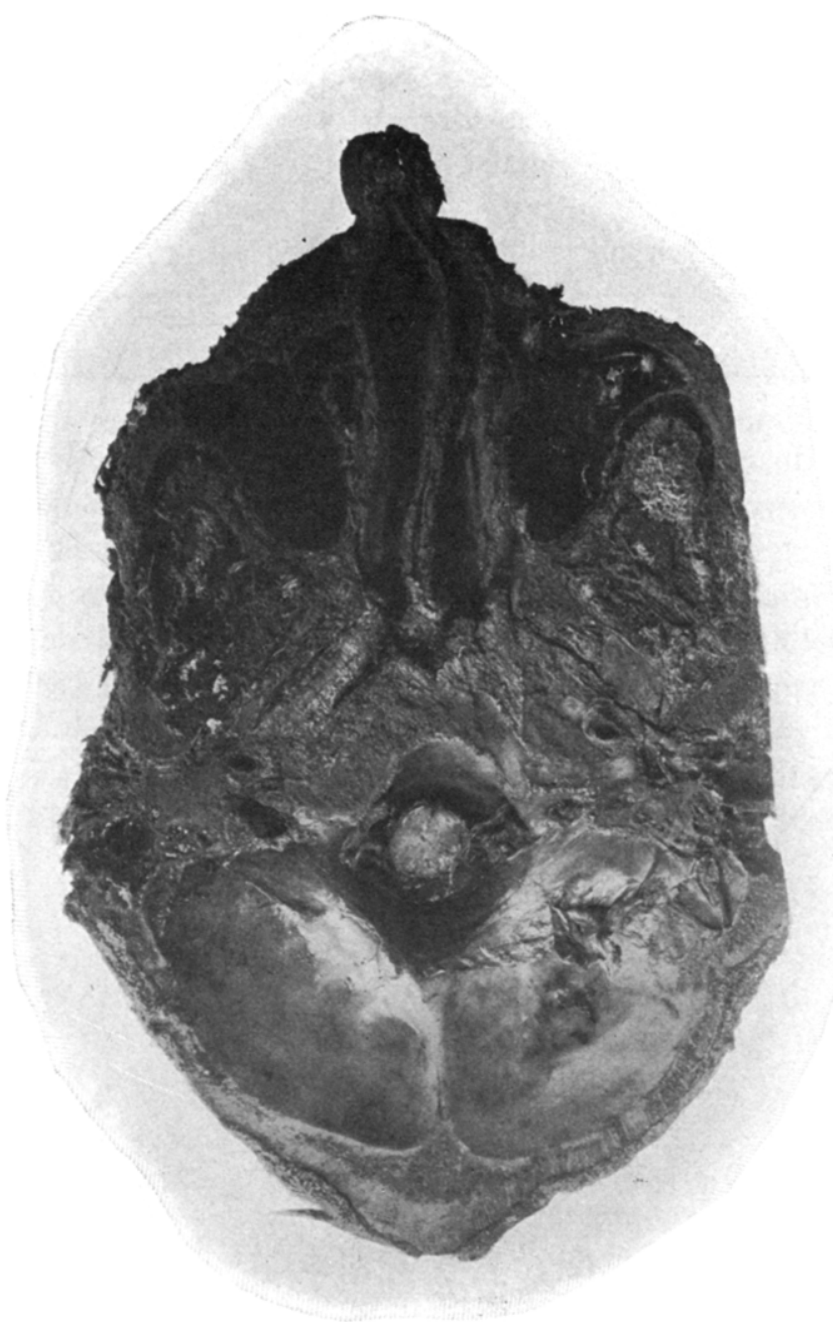

Fig. 14.-Showing nasal septum bent from before backward.

rigidity and the intermaxillary rubber would not have a tendency to displace the labial arch as it would if they were attached to the labial arch. The combination of the labial arch with the finger spur, as used by Lourie, employed in conjunction with the roof plate, as used by Richardson, gives a very ideal appliance for the treatment of patients suffering from deformed nasal cavities, narrow arches, and high palates, in which it is desirous to move the teeth bodily 
by pressure on the alveolar plates and produce as great a change in the nasal cavity as is possible.

In the increase of width of the dental arch and the increase of space in the nasal cavity, deflected septi are corrected provided the deflection is from above downward. Such deflections as are shown in Fig. 14, from before backwards, and which probably are the result of traumatism, are not corrected or benefited to any great extent by the correction of malocclusion. In beginning the treatment of a malocclusion, it is very necessary that a careful diagnosis of the malocclusion be made, as well as a careful examination of the nasal cavity, in order to recognize the condition of the nasal structures. Then the appliance must be selected with the idea of producing, as nearly as possible, the ideal results. While it is possible to produce a great change in the nasal cavity, care must be taken not to promise the patient too much; and to remember that only certain types of deflected nasal septi can be corrected.

It is my belief that in the majority of cases in which there is a lack of width between the lateral walls of the nose, this can be changed, the nasal cavity caused to increase to its proper size, and a great benefit produced, so far as respiration is concerned, provided the proper treatment is instituted. In a large number of nasal deformities there is nothing which offers so much benefit as the proper correction of the malocclusion with the idea of producing a normal nasal development. 